\title{
Design and Evaluation of Parking Position Detection with Human Cooperation for Automatic Parking
}

\author{
Yuta Abe ${ }^{\mathrm{a}}$, Takeki Ogitsu ${ }^{\mathrm{b} *}$, Hiroshi Mizoguchi ${ }^{\mathrm{a}}$ \\ aTokyo University of Science, 2461, Yamazaki, Noda, Chiba, 278-8510, Japan \\ ${ }^{\mathrm{b}}$ Gunma University, 1-5-1, Tenjin-cho, Kiryu-shi, Gunma, 376-8515, Japan \\ *Corresponding Author: ogitsu@ gunma-u.ac.jp
}

\begin{abstract}
Whenever we ride in an automobile, we must park it. Then, we want to avoid that very troublesome act if possible because it requires a certain amount of driving skills.

In recent years, the parking assist system is becoming partially implementation. This conventional system mainly recognizes the parking space surrounded by the lane. However, this conventional system is merely driving support system for supporting safety driving corresponding to the level of a driving technique of a driver. The automatic parking system have been studied previously. However, that system have an issue to depend on development of infrastructure at parking lots to detect the parking space, such as cameras and sensors or the communication technology.

Then, the authors suggest the new automatic parking system that drivers indicate parking position from the outside a car and assist the environmental recognition of the car to allow a car to park semi-automatically. This new system is another approach to park an automobile with human cooperation. Specifically, the driver parks using an onboard camera and the indication device which indicate parking position. An onboard camera detects the indication device, therefore a vehicle detect parking position. The method to detect indication device is based on HSV-colored conversion and Hough transformation. The method to calculate between the parking position and the vehicle is based on onboard camera.
\end{abstract}

Assuming some real parking positions, we evaluated making the vehicle detect parking positions using the method that we proposed.

These results show enough detection to park possible and possibility of the proposed system implementation.

Keywords: automobile, automatic parking, detection.

\section{Introduction}

Automobiles, unlike public transportation such as a train, can move to a destination without the limitations of path and time. Vehicles are widely driven because of this convenience. However, many difficulties may be encountered while driving. Among these difficulties, stopping the vehicle at a designated space or reversing the vehicle is troublesome, especially for inexperienced drivers or elderly drivers. Accordingly, research and development on driving assistance systems for parking has been conducted ${ }^{(1-5)}$. In these studies, a vehicle-mounted camera is typically used in the system to detect a white line that assists in parking. However, these systems cannot work in the absence of a white line.

Furthermore, these driving assistance systems are merely used to assist drivers; the drivers have to park the vehicle by operating the foot pedals of the vehicle. Fully automatic parking systems have been studied and developed; however, such systems require additional equipment, such as cameras and sensors, in the parking $\operatorname{lot}^{(6)}$.

This study proposes an automatic parking system that eliminates the need for additional equipment in the parking lot. In this system, the driver cooperates with the vehicle through an onboard camera. The driver instructs the vehicle to park in a particular space by using an indicating device; thus, additional equipment is not required in this system. In this paper, we describe the method and evaluate the detection of a parking space by a vehicle by using the indication device and the onboard camera. 


\section{Design of Parking Space Detection System using Human cooperation}

\subsection{Automatic Parking System with Human Cooperation Concept}

The concept behind the proposed system is that the vehicle can park automatically by using two visible light cameras. In addition, unlike conventional driver assistance systems, automatic operation of the vehicle is possible by using the proposed system. It is not necessary for a driver to operate the foot pedals of the vehicle.

Figure 1 shows the concept of the proposed system. In the proposed system, the driver indicates a parking space to a vehicle by using an indicating device. The vehicle recognizes the indicated parking space and parks in the indicated parking space. This paper describes the results of

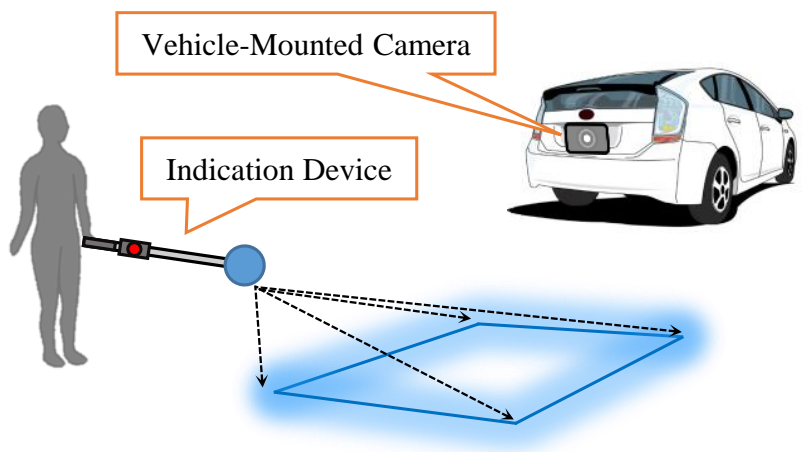

Fig. 1. Concept of the Proposed System.

the vehicle detecting the parking space and the calculated distance.

\subsection{System Configuration}

Figure 2 shows the flowchart of the proposed system. It is important that the vehicle-mounted camera recognizes the parking space and the distance to the parking space is measured. In this paper, we call the device to indicate parking space the indication device. A conceptual view of the indication device is shown in Figure 3. A marker is attached

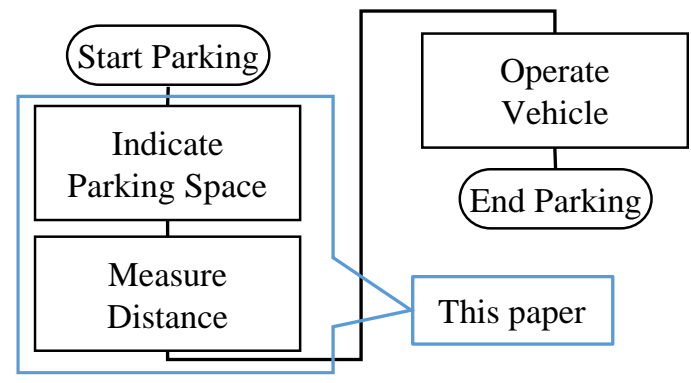

Fig. 2. System Flowchart. to the indication device such that the vehicle-mounted camera can recognize the device. In addition, buttons for sending signal to the vehicle are included in the device. Furthermore, two generic visible light cameras are used in addition to the onboard camera. The cameras are used to perform stereo vision image processing in order to measure the distance to the parking space.

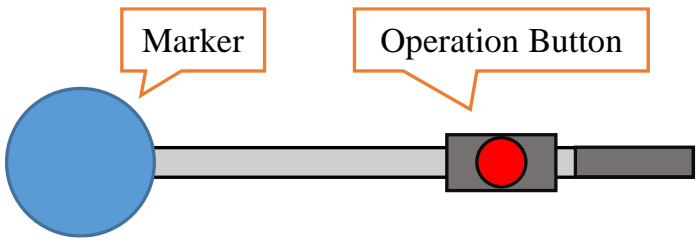

Fig. 3. Concept of Indication Device.

\subsection{Image Processing Algorithm}

The three main stages in an image processing algorithm are as follows: (1) recognizing the indication device, (2) measurement of distance by using stereo vision, and (3) detection of the marker over a wider range by using a PanTilt unit.

(1) Recognizing the indication device

In order to park, the vehicle must recognize the indication device with the help of the vehicle-mounted camera. In this study, the marker attached in the indication device was recognized using circle detection by Hough transform ${ }^{(7)}$. The detection of the center coordinates of the marker is possible by using circle detection of Hough transform. The circle candidates are produced by "voting" in the Hough parameter space and then select the local maxima in a so-called accumulator matrix. In a two dimensional space, a circle can described by:

$$
(x-a)^{2}+(y-b)^{2}=r^{2}
$$

where $(a, b)$ is the center of the circle, and $r$ is the radius. Thus, as the circles can be represented by using three parameters, which represent the center and radius of the circle, as shown in Figure 4.

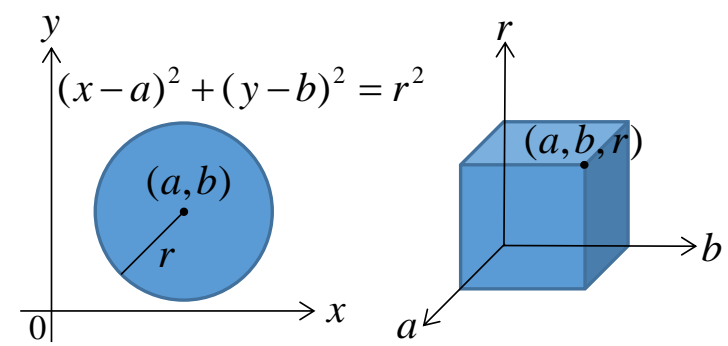

[a] xy Image Space [b] abr Parameter Space

Fig. 4. The Parameter Space of the Circle. 
In 3D space, the circle parameters are identified by intersection of many conic surfaces. The accumulator matrix was introduced to detect that intersection in parameter space. First, it is necessary to divide the parameter space into "bucket" using a grid and to make accumulator matrix that is according to a grid. The parameters of accumulator matrix show the number of circle corresponding grid cell in parameter space. The number of circle is also "voting number". First, each parameters of accumulator matrix is zero. Second, we calculate circle in parameter space and increase voting number. This process is called "voting". Voting is carried out for parameter spaces $a, b$, and $r$ for all the circle candidate pixels, and the parameters of the circle can be determined by examining the voting results ${ }^{(8)}$. In this study, in order to increase the accuracy of Hough transform, we performed two intermediate processing steps. In one step, "salt-and-pepper noise is removed by processing, using expansion and contraction. The other step involves threshold processing. This processing method either processes the threshold values to zero or to the specified value.

(2) Measurement of distance by using stereo vision

Image stereo vision data is obtained using horizontally offset cameras (stereo camera).

Based on these data, it is possible to calculate the distance. Using the triangulation method to determine the difference in appearance between objects viewed through the right and left cameras (parallax), it is possible to determine the distance to the object. A conceptual diagram of the triangulation method is shown in Figure 5. The cameras (A, B) baseline is $\mathrm{L}$ and object $\mathrm{P}$ distance is $\mathrm{D}$. Thus, the object $\mathrm{P}$ is described by the following equation:

$$
D=\frac{L \sin (\alpha+\beta)}{\sin \alpha \sin \beta}
$$

The triangulation method detects the circular markers of the indication device in each image from the image pairs obtained by the camera. Using triangulation from the center coordinates of the circle in each image, the distance between

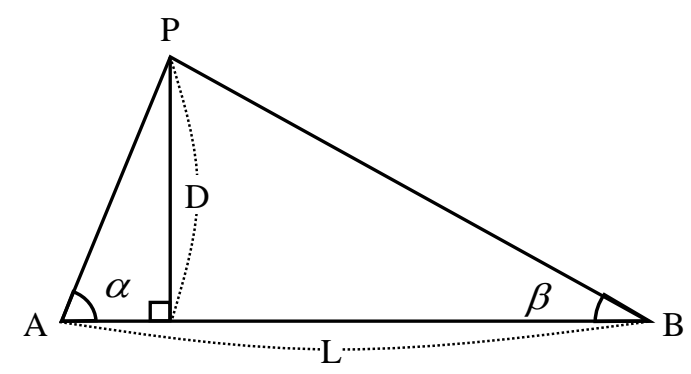

Fig. 5. Conceptual Diagram of Triangulation Method. the vehicle camera and indication device is calculated. In this study, it does not matter positional relationship in the vertical direction of the vehicle when instructing the parking position. Therefore, it calculates the position by using only the horizontal direction of the vehicle.

(3) Detection of the marker over a wider range by using a Pan-Tilt unit

In this study, the vehicle was equipped with an on-board camera on a Pan-Tilt unit. The platform can be electronically controlled. By controlling the attitude of the Pan-Tilt unit, it is possible to detect the marker over a wider range.

\section{Experiment}

In this study, in order to validate the recognition method and position measuring method of the indication device, an outdoor measurement experiment was performed. In this experiment, instead of an actual person, a tripod on which a marker was attached indicated the parking space.

\subsection{Experimental Conditions}

In the experiment, two USB cameras installed in a small electric vehicle measured the distance to the parking space, which was indicated by the indication device. The apparatus used in the experiment is shown in Figure 6. As shown in Figure 6, two USB cameras were mounted on a Pan-Tilt unit with an aluminum frame at a height of $0.5 \mathrm{~m}$ from the ground. Baseline length was set to $45.3 \mathrm{~mm}$. The USB camera used in this experiment had a view angle of $77^{\circ}$ and a resolution of 1920 x 1080 pixel.

In the evaluation method, a laser scanner (LMS100 manufactured by SICK Inc.) mounted in the front of the vehicle measured the distance. The tripod fitted with the marker was attached to a reflective plate for the laser scanner to measure the distance D from the laser scanner to the reflector. We assumed three parking spaces for the vehicle as shown in Figure 7, and we measured the four corners for each parking space. The size of the parking space was assumed to

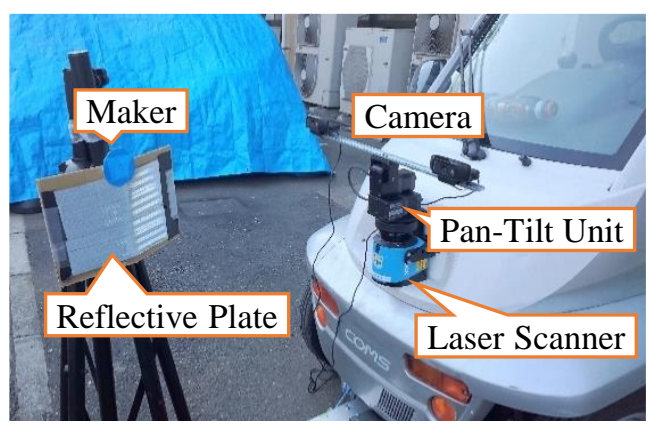

Fig. 6. Experimental Environment. 
be the same as the small electric vehicle, whose width and length were $1 \mathrm{~m}$ and $3 \mathrm{~m}$, respectively. We measured the 12 points of $\mathrm{a} \sim 1$ and evaluated.

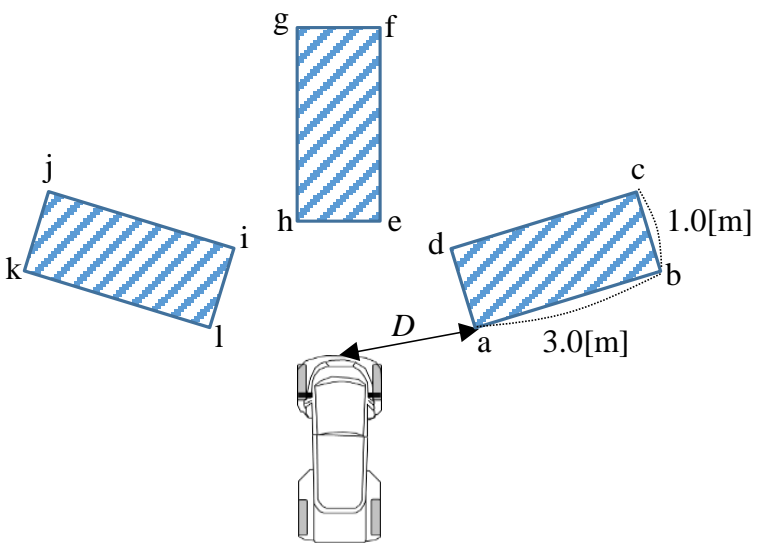

Fig. 7. Three Parking Space and Sampling Points.

\subsection{Experimental Results}

The measurement results obtained using the vehiclemounted camera and laser scanner are shown in Figure 8.

As shown in Figure 8, the minimum error in the measured distance was $0.01 \mathrm{~m}(0.23 \%)$ and the maximum error was $0.29 \mathrm{~m}(4.72 \%)$.

Therefore, the vehicle-mounted camera recognizes the parking space indicated by the indication device, and thus the proposed method is indeed effective for detecting a parking space.

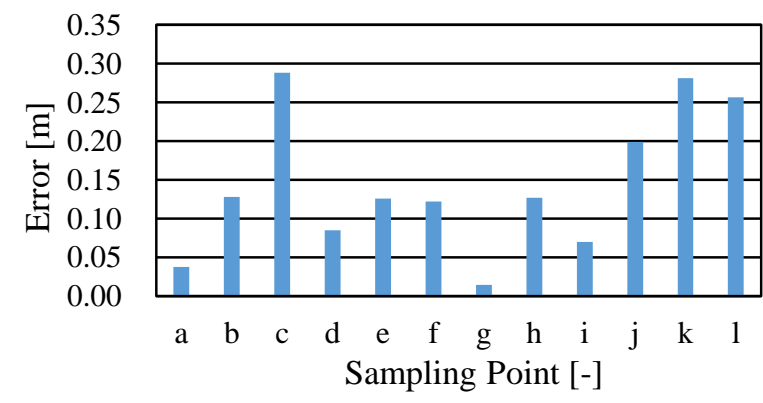

Fig. 8. Experimental Results.

\section{Conclusions}

In this paper, in order to eliminate the need for additional equipment in a parking lot, the design of a novel parking space detection system that involves human cooperation for automatic parking is proposed and evaluated. An experiment was conducted in outdoor environments by assuming three parking spaces to validate the effectiveness of the proposed system.
The results confirmed the detection of the parking space, which is indicated by the indication device, by the vehiclemounted camera; the minimum error distance was confirmed to be $0.02 \mathrm{~m}$, while the maximum error distance was $0.29 \mathrm{~m}$. These results indicate that the proposed system is effective for automatic parking.

Future studies will involve the application of the automatic parking system to the automatic parking of a small car.

\section{References}

(1) Shinji Tanaka, Yoshihiro Suda, Hiroshi Makino, and Takayuki Hirasawa : "Research and Development of Parking ITS", Proceedings of the 9th ITS Symposium 2010, 2010

(2) Nawaz Sarfraz, Christos Efstratiou, and Cecilia Mascolo : "Parksense: A smartphone based sensing system for on-street parking", Proceedings of the 19th annual international conference on Mobile computing \& networking. ACM, pp. 75-86, 2013

(3) Choeychuen Kairoek : "Automatic parking lot mapping for available parking space detection", Knowledge and Smart Technology (KST), 2013 5th International Conference on. IEEE, pp. 117-121, 2013

(4) Jung Ho Gi, Dong Suk Kim, and Pal Joo Yoon : "Parking slot markings recognition for automatic parking assist system", Intelligent Vehicles Symposium, 2006 IEEE., pp. 106-113, 2006

(5) Klaus-Peter Döge and Jürgen Krimmling : "Experiences with Video-Based Parking Space Surveillance", Proceedings of the 19th ITS World Congress, 2012.

(6) Manabu Omae, Takashi Hisamatsu, Kanji Matsushita, Shuya Sato, and Shigehiro Okada : "Development and Evaluation of Automated Vehicle Guidance Control by Infrastructure in Local Area", Proceedings of the 2015 JSAE Annual Congress (Autumn), 2015

(7) Dana H Ballard: "Generalizing the hough transform to detect arbitrary shapes", Pattern recognition, Vol.13, No. 2, pp.111-122, 1981

(8) Wu Mengjie et al. : "A Method to Detect Circle based on Hough Transform", First International Conference on Information Sciences, Materials and Energy. Atlantis Press, 2015. 